\title{
Intersecting Sensor Range Cluster-based routing algorithm for enhancing energy in WSN \\ Basavaraj G N
}

Research Scholar, Department of CS\&E, JNTUA

Email: basavarajgn@gmail.com

Jaidhar C D

Email:cdjaidhar@rediffmail.com

ABSTRACT-

The primitive goal in Wireless Sensor Network is conserving energy to overcome the constraint of limited capacity of the battery. Improving the network lifetime and reduction of energy consumption are significant factors in the routing protocol design of WSN. From the view of energy efficiency, it is important to select remaining energy, distance, cluster size, optimal CHs with an area of coverage and intersecting nodes radius. An energy efficiency clustering protocol is proposed in this paper which elects effective Cluster Heads with optimal count and active nodes for complete coverage for optimizing the network lifetime with full coverage. The simulation result shows that Intersecting Sensor Range Cluster (ISRC) based Routing Algorithm achieved $21 \%$ betterment in network lifetime as compared to present energy saving technique in WSN.

Keywords - Clustering, Routing algorithm, Cluster Head, Energy Efficiency, Wireless Sensor Network

Date of Submission: Sep 27, 2018

\section{INTRODUCTION}

$\mathrm{W}$ ireless Sensor Network (WSN) consisting of tiny sensors for monitor the events in an area. WSN comprises of dense autonomous sensor nodes of less battery energy, processing capacity, and limited storage space. So importance has to be given for reducing consumption energy of sensor node to extend the lifetime of the network [1]. A dense number of sensor nodes are divided into several clusters. Each cluster has a Cluster Head $(\mathrm{CH})$ which collects information from cluster members upon aggregation the data is sent to sink as shown in Figure 1.

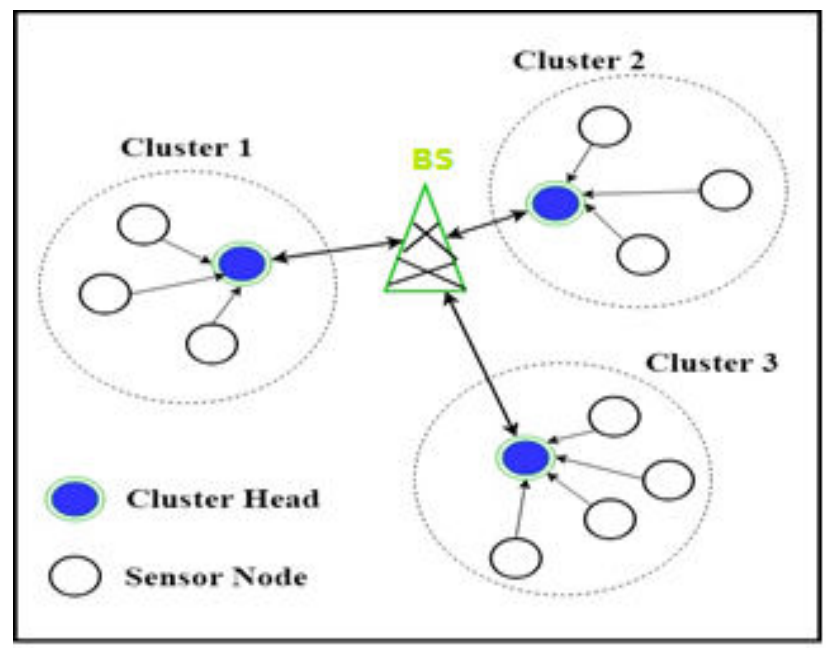

Figure 1. WSN Cluster Architecture

Modern growth in networks leads to emphasizing on energy constrained smaller, intelligent sensors. The recent development in engineering science led to a significant
Date of Acceptance: Dec 31, 2018

drive for energy conservation in the sensor network and their applications [2]. Numerous sensor nodes are distributed randomly in a harsh environment for sensing the target area, and then information is transmitted to Base Station (BS) [3].

Many routing protocols are broadly categorized into the hierarchical and flat network. Sensor Nodes (SNs) are uniform in its role and same configuration in a flat network. In a hierarchical network group, the similar cluster and selected head will communicate with BS [4]. Multihop communication is better than single hop communication. Due to the overburden of communication among nodes and their energy shed at a rapid rate, it leads to a problem called "Energy hole problem" [5]. SNs need to cover the entire area of the target which is the most significant in some of the WSN applications such as intrusion detection etc.

The unconventional method of utilizing energy leads to create an energy hole in the network. Hence, a novel energy effective algorithm for solving the problem of coverage is essential for the network. Several clustering approaches in WSN don't ensure the complete coverage of the target area and leads to the death of sensor nodes [6]. There is always a demand to maintain complete coverage despite the death of a few nodes. Additionally, few clustering techniques neglect the overlapped sensing area of SNs.

Several routing protocols overcome energy hole problem but they become obsolete in the circumstance of coverage. In contrast several coverage protocols concentrate on coverage but ignore the energy hole problem. In WSN an enhanced coverage and network, lifetime becomes important for the design of routing protocol. Hence this paper proposes new routing algorithm called Intersecting 
Sensor Range Cluster based Routing Algorithm (ISRC) for enhancing the network lifetime by ensuring energy and coverage simultaneously.

The remaining part of this work is organized as follows: Section II, comprise of the relevant work, Section III describes the research proposal and mathematical models. Section IV presents the result of simulation work along with analysis. Finally the section $\mathrm{V}$ presents a conclusion of paper with future enhancement.

\section{RELATED WORK}

Many clustering protocols are proposed in the past decade to enhance energy efficiency and to preserve coverage in WSN. The merits and demerits of typical clustering protocols and coverage preserving protocols are presented in this section.

In LEACH [7], $\mathrm{CHs}$ are elected randomly for gathering and aggregating information then send to BS directly. Each round role of $\mathrm{CH}$ is rotated among all nodes without considering the remaining energy of nodes, optimal cluster count and average distance. Average energy, minimum path loss and degree of a node are the weighing factors in a cluster based distributive algorithm for the selection of Cluster Head in the network [8]. The algorithm focuses on load balancing and on a number of clusters formations to conserve energy in the network but $\mathrm{CH}$ requires more energy for transmitting information directly to the Base Station (BS) which depletes the energy of nodes quickly. Cluster-based routing protocol [9] is developed on energyaware routing design where nodes are randomly distributed. The main objective is to maintain uniform energy consumption of each node for extending the lifetime of the network. However, maintaining coverage is uncertain in critical applications. The coverage hole is created when coverage significant node is selected as a $\mathrm{CH}$ as their energy drains rapidly.

In a mission-critical application, accuracy of monitored data fails due to coverage holes in the network. The above algorithms fail to solve energy hole problem efficiently, although the $\mathrm{CH}$ role is rotated uniformly in the WSN. A maximum number of Clustering algorithms of earlier study focus only on sensor node lifetime ignoring complete coverage of the area. In study [10] LEACHCoverage-U protocol proposed to meet the demands of coverage problems in some real-world applications. Coverage-aware parameters are considered for network coverage preserving in the Coverage-Preserving Clustering Protocol (CPCP) algorithm [11].

The selection of $\mathrm{CH}$, a relay node and active $\mathrm{SNs}$ are based on the condition of satisfying coverage preservation in the network. But, it increases the SNs computation overhead, for finding nodes location with their cost metrics.

Wang et al. proposed a Coverage-Aware Clustering Protocol (CACP) which is a modified method of selecting active nodes and $\mathrm{CH}$ for WSNs in study [12]. However, a number of CH's are not proportional which leads to the quick death of few $\mathrm{CH}$ in the network. Tao et al. [13] proposed a Flow Balanced Routing (FBR) protocol which explores the method of multi-hop for maintaining the energy and for coverage lifetime of the network. But it increases computation overhead of sensor for finding next hop before transmitting to Sink. Liu [14] proposed a scheme of average clustering called Distributed EnergyEfficient Clustering Algorithm with Improved Coverage (DEECIC). The parameters of dense deployment area, residual energy, the degree of a node are considered in DEECIC for the election of $\mathrm{CH}$. Although it enhances coverage lifetime, it does not consider the sensor activation of nodes.

Single hop transmission is inefficient in the reduction of energy consumption than multi-hop transmission [15]. In a large-scale network, multi-hop transmission is adopted in recent research [16] where aggregated data are sent across multiple $\mathrm{CHs}$ instead of sending directly to the sink. Several multi-hop transmission uses tree topology where data traffic is not shared uniformly among nodes. Moreover the important node for coverage might be exhausted early leading to decrease in coverage lifetime.

Tarhana et al. proposed a clustering protocol namely Scalable Energy Efficient Clustering Hierarchy protocol SEECH [17] studies on how to select relay nodes during clustering formation phase. Although it selects $\mathrm{CHs}$ and relays nodes in a different phase for reducing energy consumption it ignores the coverage importance.

Energy Aware Clustering Routing Protocol (EACRP) [18] uses multilayer of clusters where the width of each layer decreases near the sink. The overhead of communication is reduced but, the coverage performance parameters are ignored during the selection of $\mathrm{CH}$ in the clustering phase of the network.

In order to overcome the difficulty found in the above algorithms, an Intersecting Sensor Range Cluster based Routing Algorithm (ISRC) is proposed. Those nodes with optimal energy and prominent coverage are elected as $\mathrm{CHs}$ and active nodes. The appropriate selection of $\mathrm{CHs}$ and active sensor nodes based on cluster size, overlapping sensing range degree and cluster size consideration along with residual energy, distance, optimal cluster size count contribute more saving of energy as compared to recent existing routing algorithms. The $\mathrm{CH}$ selection is more effecting near the BS which contributes more in increasing the lifetime of the network as compared to EACRP.

\section{PRELIMINARIES \\ 3.1 NETWORK MODEL}

Some assumption in the proposed work which describes the network model is as follows

- Sensors are densely deployed randomly over an area.

- All nodes are stationary with uniform initial energy, processing, and sensing.

- A unique Identification number is assigned to each sensor without awareness of the location.

- Multi-hop data transmission to the BS

- Transmission range and Sensing range can be varied during the time of simulation. 
- The network model is based on 2 dimensional where transmission radius is two times greater than sensing radius.

- Data transfer in both directions among SNs

- Each node is well informed with their neighboring nodes.

\subsection{ENERGY MODEL}

Sensor node consists of actuators, processors, transmitter, receiver, and battery. Transmitter energy depends on the transmitter circuit which consumes energy by one bit and amplifies energy consumed by sending a message over a distance to the recipient and mode of the channel.

Transmission energy [4] is shown in equation 1.

$\mathrm{E}_{\mathrm{tr}}=\mathrm{E}_{\mathrm{a}}+\varepsilon_{\mathrm{Tx}} * 1$

$\mathcal{E}_{\mathrm{Tx}}$ - Energy required per bit of message.

The energy need to send data over free space and multipath is represented in equation (2) \& (3)

$\mathrm{E} \frac{f p}{a}=\varepsilon_{\mathrm{fs}} * 1 * \mathrm{~d}^{2} \quad$ if $\mathrm{d} \leq \mathrm{d}_{\mathrm{o}}$

$\mathrm{E} \frac{m p}{a}=\varepsilon_{\mathrm{mp}} * 1 * \mathrm{~d}^{4} \quad$ if $\mathrm{d} \geq \mathrm{d}_{\mathrm{o}}$

$\boldsymbol{E} \quad$ Represent energy spend per bit square meter.

$\mathrm{d}$ Distance between the sender node and receiver node.

fp Free space.

mp Multi path.

The total energy required for aggregating data by all SNs is represented in equation (4)

$\mathrm{E}_{\mathrm{Ag}}=\boldsymbol{\varepsilon}_{\mathrm{Ag}} * 1 * \mathrm{~N}$

$\mathrm{N}$ - Total number of nodes.

Receiver circuit requires energy for receiving the message is shown in equation (5).

$\mathrm{E}_{\mathrm{rx}}=\varepsilon_{\mathrm{rx}} * 1 * \mathrm{M}$

Where $\mathrm{M}$ is number of messages.

Gross energy consumed in one round is represented using the equation (6)

$\sum_{c=1}^{c}\left(\sum_{n=1}^{N c} \mathrm{E}_{\mathrm{tr}}+\mathrm{E}_{\mathrm{Ag}}+\mathrm{E}_{\mathrm{rx}}\right)$

Where $\mathrm{c}$ is cluster and $\mathrm{N}_{\mathrm{c}}$ is Number of clusters.

\subsection{PROPOSED ALGORITHM}

The proposed work is divided into two phases. During the first phase, clusters are formed with a selection of $\mathrm{CHs}$ for each cluster and active nodes are identified. In the second phase, Cluster Member (CM) transfer information to respective $\mathrm{CHs}$ then each $\mathrm{CH}$ aggregate the data before sending to BS.

Initially, SNs are distributed evenly on the entire area of the network. Every SNs aware of their placement and number of points covered, the density of node with residual energy, overlapping degree in each round. Every SNs announce its latest information to all nodes covered in its sensing range. As soon the messages are gathered all SNs determine Gross value $G_{v}$. The $G_{v}$ is computed using the dimension of nodes, cluster size, intersecting nodes, and remaining energy. Intersecting degree is refers to the number of nodes involved in intersecting of sensing range as shown in Figure 2

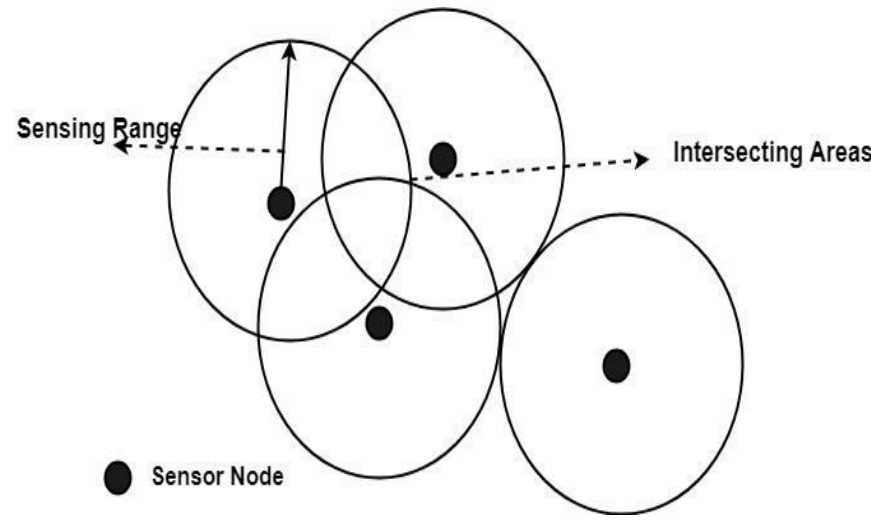

Figure 2. Intersecting Areas in WSN

In the following section, the Algorithm 1 describes the process of electing $\mathrm{CH}$ and identifying active nodes during each round of selection process in WSN.

\section{Algorithm 1}

Step 1: Start

Step 2: Randomly spread all sensors over a network with complete connectivity.

Step 3: Calculate distance among sensor nodes using RSS (Response Signal Strength)

Step 4: Calculate node dimension (node capacity of covering points), cluster size, intersecting degree, and remaining energy.

Step 5: cluster arrangement (n) [Algorithm2]

Step 6: communication phase $(\mathrm{CH}, \mathrm{BS})$ [Algorithm 3]

Step 7: If the remaining energy of a node is more than threshold energy then repeat Step4.

Step 8: Stop

Each round involves the following process. All SN's are distributed in a given area. Calculate distance among each sensor nodes. Find node density $\mathrm{N}_{\mathrm{d}}$, degree of overlapping $\mathrm{D}_{\text {olp }}$ and degree of node $\mathrm{D}_{\mathrm{sn}}$. Compare remaining node energy Er > ET then node is allowed for the subsequent round. Net valued added parameter equation is $\mathrm{N}_{\mathrm{v}}=\mathrm{E}$ $\mathrm{V} 1+\mathrm{D}_{\mathrm{sn}} \mathrm{V} 2+\mathrm{D}_{\mathrm{olp}} \mathrm{V} 3+\mathrm{N}_{\mathrm{d}} \mathrm{V} 4$. Nodes with an optimal value of $\mathrm{N}_{\mathrm{v}}$ are elected as active nodes for the given area. 
Each active node send packets within the radius of sense. The minimum distance node to the sink and optimal $\mathrm{N}_{\mathrm{v}}$ value nodes within clusters are elected for $\mathrm{CHs}$. The $\mathrm{CH}$ informs its selection to all its cluster nodes. During intra cluster communication data transfer is single hop but during inter-cluster transmission, it is multihop.

The cluster formation is presented in Algorithm 2 as follows:

\section{Algorithm 2 Function cluster arrangement (n)}

Step 1: Start

Step 2: Initialize energy parameters of nodes and assign a unique number and update the information.

Step 3: Compute $\mathrm{N}_{\mathrm{V}}=\mathrm{E} \mathrm{V1}+\mathrm{D}_{\mathrm{sn}} \mathrm{V} 2+\mathrm{D}_{\mathrm{olp}} \mathrm{V} 3+\mathrm{N}_{\mathrm{d}} \mathrm{V} 4$

Step 4: Identify active nodes in the network

Step 5: Find the distance between each node to sink

Step 6: Compare remaining energy with threshold energy and elects cluster head.

Step 7: Send a broadcast message to cluster members.

Step 8: Stop

The values of V1, V2, V3, and V4 are $0.5,0.1,0.75,0.85$ depends on different applications.

\section{Algorithm 3 Function communication phase (CH,BS)}

Step 1: Start

Step 2: Find the $\mathrm{CH}$ distance to Base Station

Step 3: Select $\mathrm{CH}$ with minimum value $\mathrm{N}_{\mathrm{v}}$ and maximum residual energy

Step 4: Identify relay nodes

Step 5: Multihop communication to Base station

Step 6: Stop

Entire area is partitioned into equal distance points and SN radius covers points. The net value $\mathrm{N}_{\mathrm{v}}$ of each node is calculated and whichever is highest is elected as active node and the rest are sleeping nodes.

The degree of overlapping involves a number of SNs and also the covered area of neighboring SNs within sensing range in selecting active nodes. Another energy parameter called the cluster size is considered for each round of selecting the $\mathrm{CH}$ in the network. Thus ISRC reduces the overall consumption of energy and increases the lifetime of the network.

Each member node sends the data directly to $\mathrm{CH}$ during intra cluster operation while the relay node is chosen on the shortest path within sensing ranges of $\mathrm{CHs}$. Thus the load of process aggregation and sending process is distributed equally around the area without creating an energy gap and improves the lifetime of WSN.

\section{RESULT}

The performance of ISRC is evaluated using NS2.34 Tcl network simulator and compared with Energy Aware Clustering Routing Protocol (EA-CRP). The analysis of result includes the residual energy of $\mathrm{CHs}$ vs. time, coverage lifetime with time, number of dead nodes with time, network residual energy with time and number of $\mathrm{CH}$ alive with respect to time. The coverage lifetime refers to SN point's coverage time in the network. Network lifetime represents duration from the beginning to till the minimum nodes die in the network.

Evaluation of results is done under energy and coverage network parameters. Table 1 shows the list of parameter and values used in the simulation.

\begin{tabular}{|l|l|}
\hline \multicolumn{1}{|c|}{ Parameter } & \multicolumn{1}{c|}{ Value } \\
\hline Network Size & 600 \\
\hline Monitoring area & $100 \mathrm{Square}$ Meters \\
\hline Base Station location & $(50,50)$ \\
\hline $\begin{array}{l}\mathrm{T}_{\mathrm{X}} / \mathrm{R}_{\mathrm{x}} \text { electronics } \\
\text { constant }\end{array}$ & $50 \mathrm{~nJ} / \mathrm{bit}$ \\
\hline Amplifier constant & $10 \mathrm{pJ} / \mathrm{bit} / \mathrm{m} 2$ \\
\hline CH energy threshold & $10^{-4} \mathrm{~J}$ \\
\hline Packet size & $20 \mathrm{bytes}$ \\
\hline Packet rate & $1 \mathrm{packet} / \mathrm{s}$ \\
\hline Sensing range & $13 \mathrm{~m}$ \\
\hline Cluster radius & $28 \mathrm{~m}$ \\
\hline Initial energy (Eint $)$ & $1 \mathrm{Joules}$ \\
\hline Transmission range & $30 \mathrm{~m}$ \\
\hline
\end{tabular}

Table 1. Parameter and values of simulation

\subsection{TIME WITH RESIDUAL ENERGY OF CH'S}

The residual energy of $\mathrm{CH}$ is the remaining energy at the defined point of operation in the network. The ISRC residual energy of $\mathrm{CHs}$ is $14 \%$ more than EA-CRP. Figure 3 represents the network lifetime(s) versus residual energy (j) of CHs.

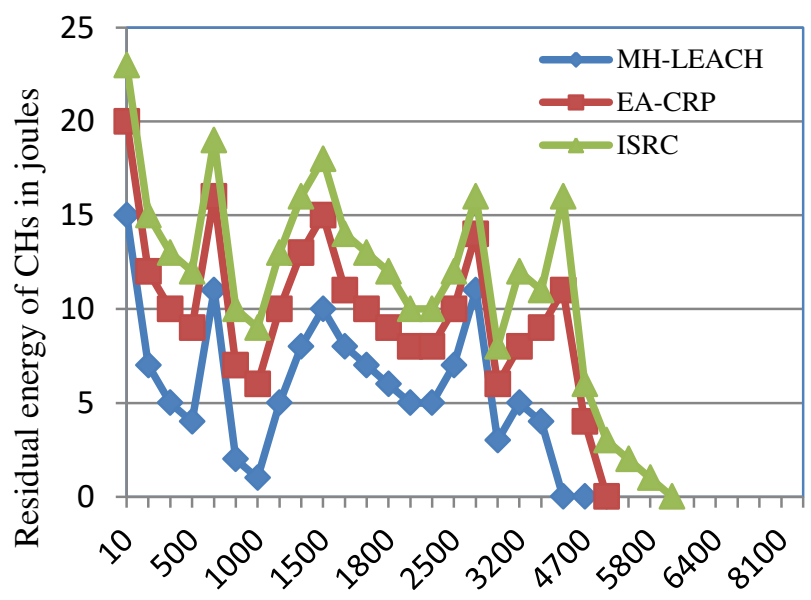

Time in seconds

Figure 3. Time(s) vs. Residual Energy of CHs

\subsection{TIME VS. COVERAGE LIFETIME}

Figure 4 represents the comparison analysis amount MLEACH, EA-CRP, and ISRC routing protocol design. 
ISRC elects optimal $\mathrm{CHs}$ and the efficient alive nodes. It shows $7 \%$ increase in coverage lifetime as compared to EA-CRP.

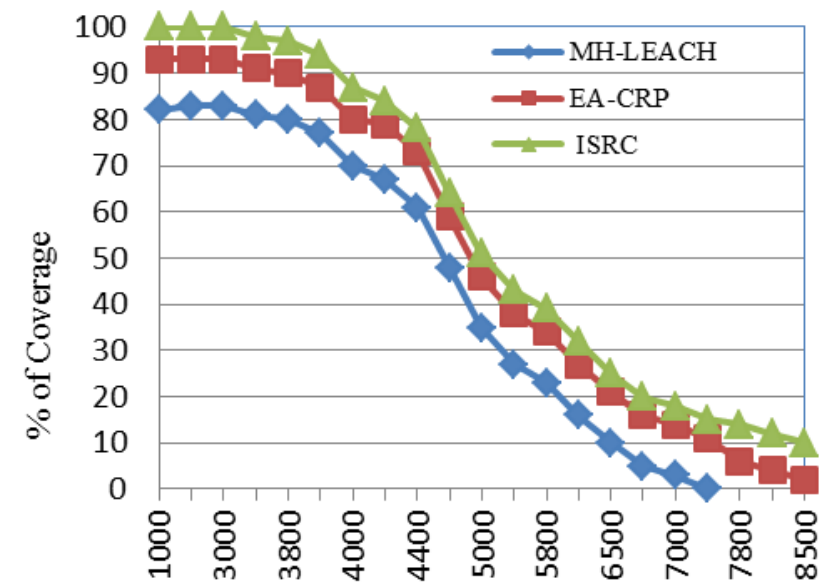

Network Lifetime(s)

Figure 4. Network lifetime with Coverage percentage

\subsection{FND, HND and LND}

ISRC performance measured in terms of First Node Die (FND) time, Half Node Die (FND) time and Last Node Die (LND) time as shown in the Figure 5.

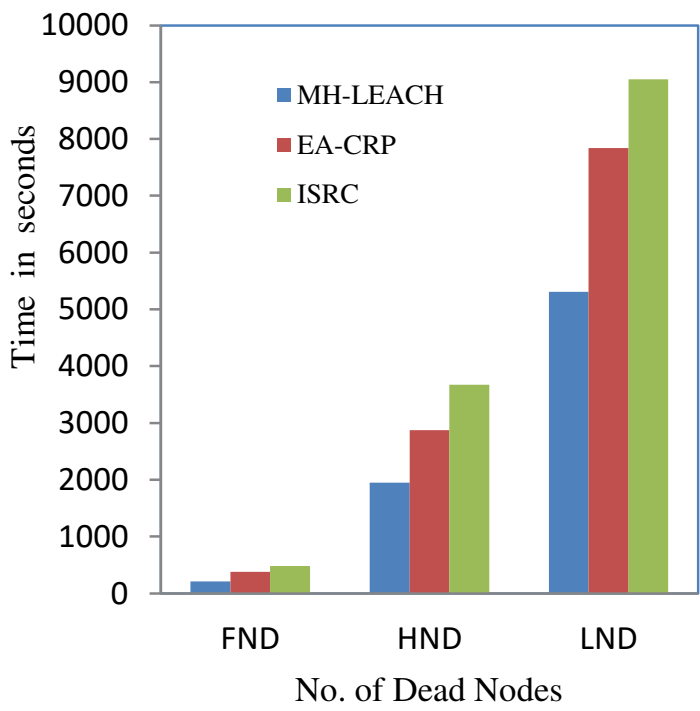

Figure 5. FND, HND and LND vs. Time
4.4 VARIED NETWORK SIZE.

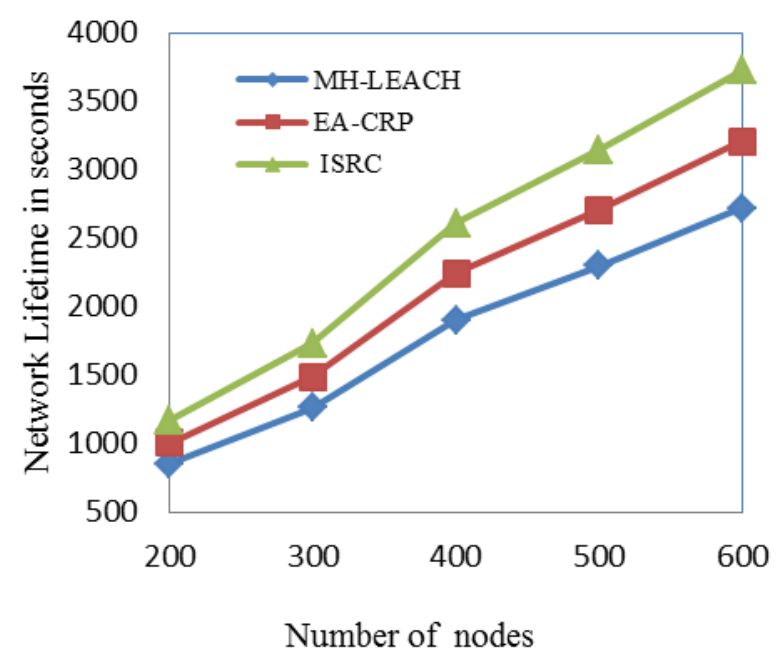

Figure 6. Number of nodes vs. lifetime(s)

The maximum number of sensors deployed is up to 600 which are varied from 200 at an interval of 100 for comparing the lifetime of the network. The methodology of the proposed model for selecting $\mathrm{CH}$ and relay node is based on intersecting range and avoids the extra energy required for communicating with the BS. It increases $20 \%$ and $13.5 \%$ more lifetime than MHLEACH and EA-CRP respectively.

\subsection{NUMBER OF CH's ALIVE WITH TIME}

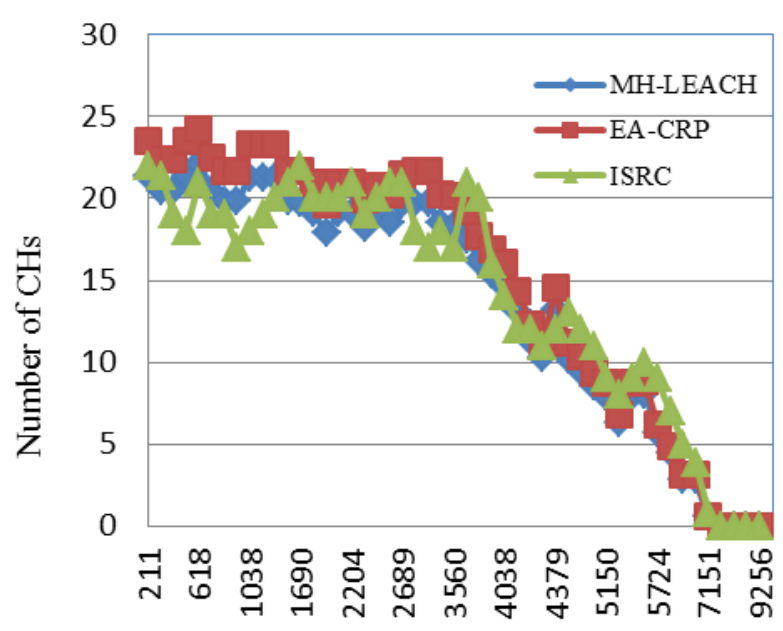

Time in seconds

Figure 7. Number of CHs with time

The total count of $\mathrm{CHs}$ alive during the network process are shown in Figure 7 . The ISRC shows a significant reduction in $\mathrm{CHs}$ count of $14 \%$ at a specific point with respect to EA-CRP. 


\section{CONCLUSION}

The objective of ISRC is to conserve battery energy using combine values of energy parameter to improve the lifetime of the network with coverage. Energy parameters include remaining energy, distance, node coverage dimension, cluster size, intersecting sensor ranges and placement of sensor nodes for reducing the consumption of energy and for enhancing the lifetime of WSN. A novel energy based routing design called as Intersecting Sensor Range Cluster based Routing Algorithm. An optimal count of CH's and selecting active nodes is based on the sum of energy parameters which decides the lifetime of the network. The functionality of ISRC using simulation with a various parameter like increasing initial energy of nodes, variation in sensor range and a different number of sensor nodes. The simulation results shows, the proposed model achieve a significant lifetime improvement as compared to earlier routing design for energy conservation in WSN.

\section{REFERENCE}

[1] Zytoune, O., Fakhri, Y., Aboutajdine, D.: A fairly balanced clustering algorithm for routing in wireless sensor networks. Sensor Review. Emerald. 30(3), 242-249 (2010).

[2] Alkalbani, A.S., Tap, A.M., Mantoro, T.: Energy consumption evaluation in trust and reputation models for wireless sensor networks Information and Communication Technology for the Muslim World (ICT4M), Morocco, pp. 1-6 (2013).

[3] Mostafa Noorikhameneh et.al: A Survey on the Mobile Sink Hierarchical Routing Protocols in the Wireless Sensor Networks. International Journal of Advanced Networking and Applications Volume: 08 Issue: 01 Pages: 2979-2985 (2016).

[4] Haseeb, K., Bakar, K.A., Abdullah, A.H. et al. Adaptive energy aware cluster-based routing protocol for wireless sensor networks. Wireless Network, 23(2017), 1953-1966.

[5] Huang, J., Hong, Y., Zhao, Z. and Yuan, Y. An energy-efficient multi-hop routing protocol based on grid clustering for wireless sensor networks. Cluster Computing, 20(4), pp.3071-3083. (2017).

[6] Xin Gu, Jiguo Yu, Dongxiao Yu, Guanghui Wang, Yuhua Lv, ECDC: An energy and coverage-aware distributed clustering protocol for wireless sensor networks, Computers \& Electrical Engineering, Volume 40, Issue 2,Pages 384-398 (2014).

[7] Heinzelman Wendi, Chandrakasan Anantha, Balakrishnan Hari An application-specific protocol architecture for wireless microsensor networks. IEEE Trans Wireless Communication 1(4):660-70.
[8] Mahajan, S., Malhotra, J., \& Sharma, S. (2014). An energy balanced QoS based cluster head selection strategy for WSN. Egyptian Informatics Journal, 15, 189-199. [9].

[9] Yu Jiguo, Qi ,Yingying, Wang Guanghui. A clusterbased routing protocol for wireless sensor networks with nonuniform node distribution. AEÜ International Journal of Electronics and Communication 66:54-61 (2012).

[10] Tsai Yuh-Ren, Coverage-preserving routing protocols for randomly distributed wireless sensor networks, IEEE Transaction Wireless Communication, 6:1240 5 (2007).

[11] Soro, S., \& Heinzelman, W. B,Cluster head election techniques for coverage preservation in wireless sensor networks. Ad Hoc Networks, 7(5), 955-972 (2009).

[12] Bang Wang, Hock Beng Lim, Di Ma, A coverageaware clustering protocol for wireless sensor networks, Computer Networks, Volume 56, Issue 5, Pages 1599-1611 (2012).

[13] Tao, Y., Zhang, Y \& Ji, Y. Flow-balanced Routing for Multi-hop Clustered Wireless Sensor Networks. Ad Hoc Networks, 11(1), 541-554, (2013).

[14] Liu, Z., Zheng, Q., Xue, L., \& Guan, X. A distributed energy-efficient clustering algorithm with improved coverage in wireless sensor networks. Future Generation Computer Systems, 28(5), 780-790 (2012).

[15] Rajesh Krishna Panta, Issa Khalil, Saurabh Bagchi, Luis Montestruque. Montestruque, Single versus multi-hop wireless reprogramming in sensor networks. In: Proceeding of the 4th int. conf. Trident Communication (2008).

[16] Liu Zhixin, Zheng Qingchao, Xue Liang, Guan Xinping. A distributed energy-ef.client clustering algorithm with improved coverage in wireless sensor networks. Future Generation Computer System 28:780-90 (2012).

[17] Tarhani, Mehdi, Yousef S. Kavian, and Saman Siavoshi. "SEECH: Scalable Energy Efficient Clustering Hierarchy Protocol In Wireless Sensor Networks." IEEE Sensors Journal 14.11: 3944-3954 (2014).

[18] Khalid A. Darabkh, Noor J. Al-Maaitah, Iyad F. Jafar, Ala' F. Khalifeh, EA-CRP: A Novel Energy-aware Clustering and Routing Protocol in WSNs, Computers \& Electrical Engineering,(2017). 